\title{
Characterization of EuS Nanotubes in Quantum Confinement
}

\author{
A.M. Thron, ${ }^{*}$ C.S. Bonifacio, ${ }^{*}$ N. Erdman, ${ }^{* *}$ M.A. Harrison, ${ }^{* * *}$ S. Somarajan, *** J.H. \\ Dickerson, ${ }^{* * *}$ and $\mathrm{K}$. van Benthem*
}

* Department for Chemical Engineering and Materials Science, University of California, 1 Shields Ave., Davis, CA

** JEOL USA, Inc., 11 Dearborn Road, Peabody, MA

*** Department of Physics and Astronomy, Vanderbilt University, 2301 Vanderbilt Place, Nashville, TN

Europium monochalcogenides of the form $\mathrm{EuX}(\mathrm{X}=\mathrm{O}, \mathrm{S}, \mathrm{Se}, \mathrm{Te})$ have fascinating optical, magneto optical and magnetic properties. For face-centered cubic EuS, increased Curie temperatures and reversal in the remanent magnetization were observed with decreasing particles sizes, ultimately leading to quantum confinement structures.

EuS nanoparticles were synthesized by thermolysis of a molecular single source precursor. Dependent on the synthesis parameters, particles sizes ranging either between $2.0 \mathrm{~nm}$ and $10.0 \mathrm{~nm}$ (nanocrystalline region) or below $2.0 \mathrm{~nm}$ (quantum-confined region). With shrinking particle size, surface strain effects can alter the atomic arrangements and therefore the crystal structure. Since the magnetic properties of EuS are governed by the strongly bound $4 \mathrm{f}$ orbital, changes in the atomic arrangement thus leads to modified orbital overlaps between nearest neighbor lattice sites and next-nearest neighbor sites. Consequently, size-dependent optical, magnetic [1,2], and structural characteristics can been observed.

Aberration-corrected STEM analysis revealed the presence of highly crystalline face-centered cubic nanoparticles with dimensions well below $2 \mathrm{~nm}$. Figure 1 shows an annular dark field STEM micrograph with nanocrystals of various sizes within the quantim-confined region. The detection of isolated $\mathrm{Eu}$ atoms dispersed on the carbon support film indicates atomic resolution and single atom sensitivity during the STEM experiments. In many areas of the sample, non-crystalline agglomerates of Eu atoms (likely in conjunction with sulfur atoms) were observed [3].

Recently, Harrison et al. synthesized EuS nanotubes with unique optical properties due to unidirectional quantum confinement [4]. SEM, TEM and focused ion-beam microscopy confirmed hollow nanotube morphologies (Figure 2) with wall thickness of the order of 2-5 nm. The rolled-up sheets of EuS were found to be poly-crystalline with grain sizes as small as $10-15 \mathrm{~nm}$. At the time of writing, preliminary data suggest an equilibrium length and diameter of the nanotubes. Furthermore, strain within the sheets seems to cause rupturing of the nanotubes for stress relaxation (cf. Figure 2b). Currently, selected area diffraction and analytical STEM experiments are carried out for more detailed evaluations of atomic and electronic structures as well as local chemistry and oxidation states.

[1] M. L. Redigolo et al., Appl. Phys. Lett. 89, 222501 (2006).

[2] F. Zhao et al., Small 2, 244 (2006).

[3] M. L. Redigolo et al., Mat. Chem. Phys. submitted (2009).

[4] M. A. Harrison et al., Materials Letters, in preparation (2009). 
[5] Financial support came from the University of California at Davis (A.M.T., C.S.B. K.v.B.) and from the U.S. Department of Energy, Basic Sciences, Division of Materials Science and Engineering (M.A.H. and J.H.D). The authors further acknowledge assistance from G. McMahon (Boston College) and S.J. Pennycook (ORNL).
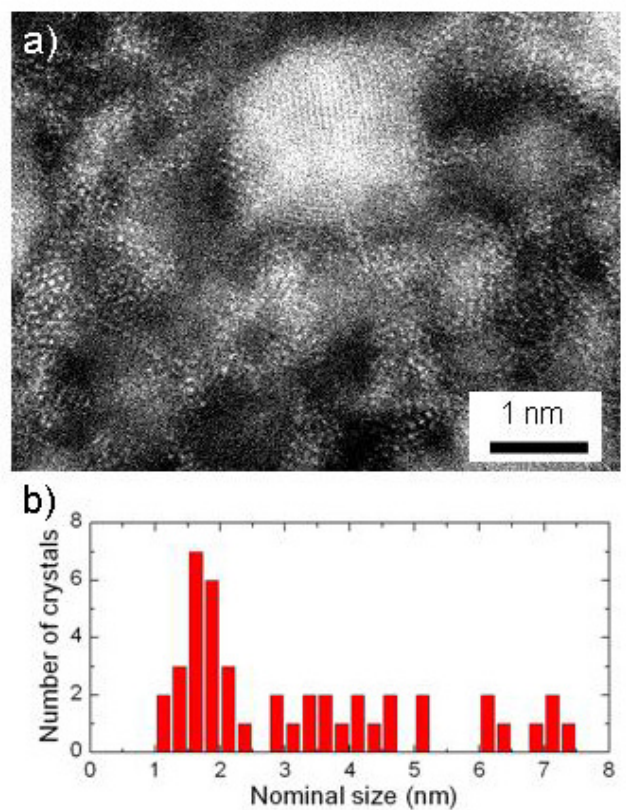

Figure 1: (a) Annular dark field STEM image of EuS nanocrystals and (b) their size distribution (adapted from [3]).
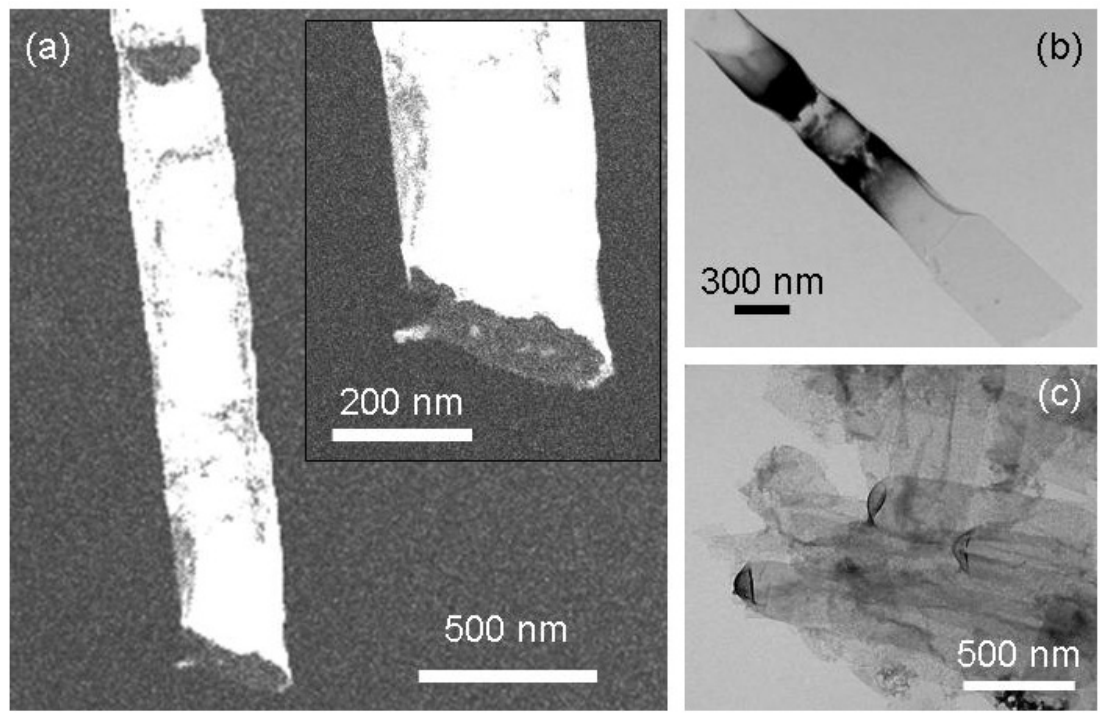

Figure 2: (a) Annular dark field STEM image of a EuS nanotube. The inset demonstrates the poly-crystallinity. (b) STEM image recorded at $30 \mathrm{keV}$ using a Jeol JSM7600 FESEM.

(c) High resolution TEM micrograph displaying a bundle of EuS nanotubes. 